Economía Teoría y PrÁctica • Nueva Época, número 47, julio-diciembre 2017, pp. 167-190, http://dx.doi.org/10.24275/ETYPUAM/NE/472017/Gonzalez

\title{
Regulación de comisiones en cajeros automáticos. Análisis de la reforma: caso México. El caso de bancos con redes de distinto tamaño*
}

\author{
Regulation of Commissions in ATMs Analysis \\ of the Reform in Mexico: The Case of Banks \\ with Different Size Networks
}

\author{
Pedro Isidoro González Ramírez** \\ Juan Carlos Neri Guzmán***
}

\begin{abstract}
RESUMEN
Partiendo de un escenario asimétrico en el tamaño de red, se analizan los efectos de la regulación establecida por el Banco de México en 2010 en comisiones por transacciones interbancarias en cajeros automáticos. Se estudia el efecto de la regulación sobre aquellos bancos con redes de cajeros pequeños y por lo tanto con mayores proporciones de retiros de clientes propios en cajeros ajenos. Se observa que las comisiones por transacciones interbancarias se mantienen relativamente sin cambios. Los resultados revelan que la regulación aumenta los precios por apertura de cuenta, al prohibir el cobro por transacciones a usuarios propios en cajeros propios. Finalmente, el aumento de precios por apertura de cuenta es menor para el banco con menos cajeros instalados, por lo que la regulación favorece a los bancos pequeños.
\end{abstract}

Palabras clave: Cajeros automáticos, cuotas de intercambio, comisión por uso de cajero ajeno, cuota de sobrecargo, cobro directo.

Clasificación JEL: D40, G21, L51.

\begin{abstract}
In the context of an asymmetric scenario in network size, we analyze the effects of the regulations established by the Mexican Central Bank in 2010 on fees for interbank transactions at ATMs. We studied the regulations' impact on banks with small ATM networks and commensurately higher percentages of withdrawals by their customers in non-proprietary ATMs. We noted that fees for interbank transactions have remained relatively unchanged. The results show that the regulations have driven up account opening costs by forbidding banks to charge their account holders for transactions on proprietary ATMs. Finally, the increase in cost of opening an account is less for banks with fewer installed ATMs, which means that the regulations favor smaller banks.
\end{abstract}

Keywords: ATMs, exchange fees, commission for use of cashier's account, charge of surcharge, direct charge.

JEL Classification: D40, G21, L51.

* Fecha de recepción: 03/02/2015. Fecha de aprobación: 11/01/2017.

** Facultad de Economía, Universidad Autónoma de San Luis Potosí, México. Correo electrónico: pedro.gonzalez@uaslp.mx. ORCID.0000-0002-0763-954X

*** Universidad Politécnica de San Luis Potosí, México. Correo electrónico: carlos.neri@ upslp.edu.mx. ORCID 0000-0002-2711-6797 


\section{INTRODUCCIÓN}

Desde que los cajeros automáticos fueron introducidos por primera vez hace poco más de 40 años, se han convertido en un elemento fundamental de las operaciones diarias de cualquier consumidor. Los cajeros automáticos ofrecen beneficios significativos tanto a los bancos como a sus usuarios. A los bancos les permite importantes economías de escala al automatizar transacciones de caja y a los usuarios les facilita el acceso a efectivo en tiempos y lugares distintos a los de la sucursal bancaria; beneficios que son multiplicados cuando los bancos deciden compartir sus redes de cajeros, permitiendo a los usuarios realizar transacciones en cajeros distintos a los del banco emisor de su tarjeta.

En México, de acuerdo al esquema tradicional vigente hasta mayo del 2010, cuando algún usuario realizaba transacciones en cajeros distintos al banco emisor de su tarjeta, podía pagar hasta dos comisiones: una al banco emisor de su tarjeta conocida como "comisión por uso de cajero ajeno" y otra al banco operador del cajero denominada "cuota de sobrecargo". Adicionalmente, el banco emisor de la tarjeta tenía que pagar una cuota de intercambio al banco operador del cajero para compensarlo por los costos de instalación y mantenimiento. ${ }^{1}$ En este caso, el pago final para el usuario por la transacción interbancaria era igual a la suma de las comisiones por uso de cajero ajeno y sobrecargo. ${ }^{2}$

El "doble pago" por parte de los consumidores hacía que las transacciones interbancarias fueran altamente costosas, por lo que tanto reguladores como consumidores cuestionaban la racionalidad de la cuota de sobrecargo dado que ya existía un sistema de cuotas de intercambio que compensaba a los operadores de cajeros por procesar este tipo de transacciones. Los operadores de cajeros se justificaban argumentando que la cuota de sobrecargo les generaba ganancias directas que proveían incentivos para incrementar su tamaño de red, lo cual beneficiaba a los consumidores.

En mayo de 2010, entró en vigor un paquete de reformas diseñadas por el Banco de México para mejorar la competencia y la eficiencia en el mercado de cajeros automáticos. Dichas reformas modificaron estos aspectos que eran alta-

${ }^{1}$ En México la comisión por uso de cajero ajeno variaba entre 17 y 20 pesos, e incluía el costo de la cuota de intercambio ( 7.25 pesos) que pagaba el banco emisor de la tarjeta al banco operador del cajero. La cuota de intercambio era uniforme para todos los cajeros y bancos. La cuota de sobrecargo la establecían alrededor del 18 por ciento de los cajeros y oscilaba entre 8.7 y 26 pesos.

${ }^{2}$ Adicionalmente, el cajero automático no informaba a los usuarios de otros bancos el total de la comisión por la transacción interbancaria, ya que la comisión por uso de cajero ajeno no era la misma en todos los bancos. El monto de la comisión era conocido por el usuario hasta que recibía su estado de cuenta, varios días después de haber realizado la transacción. 
mente cuestionados por el público en general y por las autoridades encargadas de competencia. Las modificaciones más destacadas de la reforma fueron las siguientes: $i$ ) transparencia de la información: antes de que se autorice la operación, ya sea retiro de efectivo o consulta de saldo, se deberá mostrar al usuario el importe total de la comisión; ii) se estableció que el cobro de comisiones por operaciones interbancarias sólo podrá realizarse por el operador del cajero. Asimismo, se estableció que el banco emisor de la tarjeta no puede cobrar comisiones a sus usuarios por acudir a un cajero de la competencia; iii) se estableció que los bancos no pueden cobrar comisiones a sus usuarios cuando éstos realizan retiros de efectivo o consultas de saldo en sus cajeros. Adicionalmente, los bancos operadores de cajeros deben pagar una comisión (la cual se denomina cuota de intercambio inversa) a los bancos emisores cada vez que uno de sus clientes acuda al cajero. ${ }^{3}$

El esquema original facilitaba a los bancos con una red de cajeros pequeña ofrecer a sus usuarios el acceso a cajeros automáticos de la competencia a un costo relativamente bajo al poder subsidiar este tipo de transacciones, lo cual era posible dado que la cuota de sobrecargo era poco común. En un mercado como el mexicano donde, de acuerdo con datos del Banco de México, los cinco bancos más grandes del país poseen el 79 por ciento de los cajeros, los cuales a su vez concentran el mayor número de cuentas con tarjetas de débito, 87 por ciento, ${ }^{4}$ resulta relevante entender los impactos de la regulación sobre los bancos con menor red de cajeros que, parecería, son los principales afectados con el nuevo esquema de comisiones (cobro directo) al no poder compensar a sus usuarios cuando estos realicen transacciones en cajeros ajenos. Adicionalmente, los bancos con mayor número de cajeros pueden utilizar el cobro directo como un mecanismo para atraer clientes de bancos pequeños que desean evitar los altos cobros por transacciones interbancarias.

Motivado por la situación anteriormente descrita, en el presente artículo se busca evaluar el impacto de la regulación establecida por el Banco de México desde una perspectiva teórica. Partiendo de un escenario asimétrico en el tamaño de red, se pretende analizar el efecto de la regulación sobre aquellos bancos con redes de cajeros pequeñas y por lo tanto mayores proporciones de retiros de clientes propios en cajeros ajenos.

${ }^{3}$ Las disposiciones $i$ ) y ii) entraron en vigor el 5 de mayo de 2010; la disposición iii) entró en vigor el 27 de julio de 2010. Fuente: Diario Oficial de la Federación, "Circular" 14/2010 y 22/2010 dirigida a las instituciones de crédito, sociedades financieras de objeto limitado y sociedades financieras de objeto múltiple reguladas.

${ }^{4}$ Reporte del Sistema Financiero junio de 2010 del Banco de México. 
En lo que respecta a la literatura sobre este tema, solamente Kaiser y Lever (2011) y González y Plata (2015) han analizado teóricamente los efectos de la regulación en México. Basados en el trabajo de Massoud y Bernhardt (2002), Kaiser y Lever (2011) analizan en particular los efectos de la prohibición a los bancos en el cobro de comisiones a clientes propios en cajeros propios (comisiones a cuentahabientes) establecida por el Banco de México. En el modelo los bancos pueden fijar tres tipos de comisiones: precios por apertura de cuenta, comisiones a cuentahabientes y sobrecargo. Los autores, para hacer manejable el modelo, eliminan del análisis comisiones por uso de cajero ajeno, cuota de intercambio e instalación de cajeros. ${ }^{5}$ De acuerdo con Kaiser y Lever (2011), prohibir las comisiones a cuentahabientes también reduce la cuota de sobrecargo, pero aumenta los precios por apertura de cuenta. El aumento en precios por apertura de cuenta es menor que la disminución en las otras comisiones provocando un aumento en el excedente de los consumidores. Sin embargo, los autores encuentran una disminución en el excedente total, la cual es absorbida por las ganancias de los bancos.

Por su parte, González y Plata (2015), a diferencia de Kaiser y Lever (2011), consideran el impacto que tiene la regulación en México sobre instalación de cajeros y comisiones por uso de cajero propio y ajeno, permitiendo la existencia de cuotas de intercambio, comisiones por uso de cajero ajeno y sobrecargo. Bajo un modelo donde los bancos compiten por depósitos y por retiros de efectivo en cajeros, los autores encuentran que, independientemente de la regulación, las comisiones por transacciones interbancarias se mantienen sin cambios. Adicionalmente, la regulación incentiva la instalación de cajeros, efecto por el cual se da una disminución en las ganancias de los bancos. Los autores concluyen que ante la prohibición en el cobro de comisiones en cajeros propios, los bancos optaron por aumentar sus precios por apertura de cuenta.

Respecto a cuotas de sobrecargo y asimetrías en el tamaño de red, en la literatura se han hecho varias contribuciones; por ejemplo, Hannan, Kiser, Prager y McAndrews (2003) analizan las condiciones bajo las cuales los bancos prefieren establecer sobrecargo. Encuentran que los bancos con mayor número de cajeros son más propensos a establecer sobrecargo. Adicionalmente, encuentran que la cuota de sobrecargo no sólo se determina en función de costos de operación, sino que sirve como una estrategia para incrementar los costos de los clientes de bancos rivales.

${ }^{5}$ En este sentido, los autores sólo analizan los efectos de la regulación respecto a la eliminación de comisiones a cuentahabientes, pero no incorporan en su análisis el efecto que tiene la eliminación de la comisión por uso de cajero ajeno y la existencia de cuotas de intercambio. 
Massoud y Bernhardt (2002) desarrollan un modelo espacial con dos bancos ubicados en cada lado de un círculo donde los consumidores se distribuyen uniformemente alrededor de éste. Los consumidores pagan una cuota fija por manejo de cuenta y comisiones por transacciones en cajeros. Los autores encuentran que los bancos grandes establecen mayores comisiones por manejo de cuenta y mayores cuotas de sobrecargo que los bancos pequeños, con el propósito de incrementar el costo por transacciones interbancarias y atraer a los clientes de bancos pequeños que desean evitar estos cobros excesivos.

Prager (2001), desde una perspectiva empírica, analiza la experiencia en Estados Unidos, antes de 1995, de los bancos pequeños en estados donde era permitida la cuota de sobrecargo con bancos similares en estados donde la cuota de sobrecargo estaba prohibida. Encontró que la cuota de sobrecargo no fue suficiente para provocar que los clientes de bancos pequeños se cambiaran a bancos con mayores redes de cajeros. Esto se debe a que los bancos pequeños en los estados donde el sobrecargo era permitido no perdieron participación de mercado con respecto a los bancos pequeños en estados donde la cuota de sobrecargo estaba prohibida.

Por otra parte, Chioveanu, Fauli-Oller, Sandonis y Santamaria (2009) examinan un modelo de dos bancos bajo un esquema de competencia espacial de Hotelling, donde los bancos se localizan en los extremos de una línea de tamaño unitario. Los cajeros automáticos están localizados exógenamente en otro espacio, que los autores definen como espacio de compras. El espacio de compra está compuesto por tiendas comerciales. Los consumidores visitan las tiendas comerciales con una probabilidad igual y exógenamente determinada. Los autores examinan, bajo un equilibrio numérico con cuotas de intercambio y comisiones por uso de cajero ajeno, los efectos de las cuotas de sobrecargo sobre comisiones e instalación de cajeros. Los autores observan correlaciones positivas entre un mayor tamaño de red con cuotas de sobrecargo, precios por apertura de cuenta y ganancias de los bancos. El resultado es congruente con estudios empíricos como el obtenido por Massoud, Saunders y Scholnick (2005), donde encuentran que una mayor red de cajeros permite a los bancos captar mayores participaciones de mercado.

Dado que no existe un modelo estándar para modelar el mercado de cajeros, se utiliza el modelo propuesto por Chioveanu et al. (2009) y González y Plata (2015). Estos modelos, a diferencia de Kaiser y Lever (2011), permiten un análisis más completo de la regulación al incorporar en el modelo: comisiones por uso de cajero ajeno, sobrecargo, comisiones a cuentahabientes, cuotas de intercambio e instalación de cajeros.

El modelo presenta las siguientes similitudes y diferencias respecto al de Chioveanu et al. (2009) y González y Plata (2015). De la misma forma se hace el supuesto de un esquema de competencia espacial de Hotelling, donde dos bancos se 
localizan a los extremos de una línea de tamaño unitario. Los consumidores realizan compras y retiran efectivo en otro espacio, al que se define como espacio de compra, pero a diferencia de Chioveanu et al. (2009) se hace el supuesto de que los bancos instalan sus cajeros de manera uniforme en el espacio de compra ya que los consumidores se distribuyen uniformemente cuando necesitan efectivo. A diferencia de Chioveanu et al. (2009) y González y Plata (2015), para poder analizar el efecto de la regulación, partiendo de asimetrías en el número de cajeros, se hace el supuesto de que el número de cajeros instalado por cada banco ya está determinado. Finalmente, el presente artículo se puede ver como una extensión del artículo de González y Plata (2015) permitiendo asimetrías en el tamaño de red. Los resultados obtenidos en el caso de simetría en el número de cajeros instalados son análogos a los obtenidos por González y Plata (2015).

Bajo un modelo duopólico en que los bancos están localizados a los extremos de un segmento de tamaño unitario, donde los consumidores se encuentran uniformemente distribuidos, se endogeneiza la elección en precios, comisiones en cajeros, así como la elección de los consumidores por un banco y el número de transacciones en cajeros propios o ajenos. Nos concentraremos en entender las implicaciones de la regulación sobre: precios, comisiones interbancarias y ganancias de los bancos. Dada la complejidad de las ecuaciones se utilizan métodos numéricos para calcular el equilibrio. Esto es, calcular el equilibrio para cada posible situación de asimetrías en el tamaño de red.

Cuando un consumidor desea realizar una transacción en algún cajero, se hace el supuesto de que no sabe exactamente su ubicación, por lo cual busca alguno para poder realizarla. Con una probabilidad exógenamente determinada encuentra un cajero; una vez ubicado el cajero, la transacción solamente puede realizarse en dicho cajero; es decir, que cambiar de ubicación es altamente costoso. ${ }^{6}$ Se hace el supuesto que dentro del espacio de compra la ubicación de los cajeros es uniforme, por lo que las densidades de cajeros determinan las probabilidades de encontrar un cajero. En los resultados se encontró que a pesar de que la regulación elimina el cobro de comisiones por uso de cajero ajeno, con el propósito de eliminar cobros excesivos, la comisión por transacciones interbancarias se mantiene más o menos sin cambios. Se observa que se da un desplazamiento de las comisiones por el uso de cajeros propios (las cuales la regulación prohíbe) a mayores precios por apertura de cuenta. En términos del excedente del consumidor, el aumento en precios por apertura de cuenta excede al beneficio obtenido por transacciones gratuitas en cajeros propios, lo que ocasiona una disminución en el excedente de los consumidores. Los

${ }^{6}$ Lo cual implica una demanda sumamente inelástica al exagerar los costos de transporte. 
resultados indican que una mayor red de cajeros permite a los bancos captar mayores participaciones de mercado, mayores precios por apertura de cuenta y mayores cuotas de sobrecargo. Finalmente, se observa que la regulación favorece la competencia al generar que el aumento en costos, por la eliminación del cobro en cajeros propios, sea mayor para el banco con un mayor tamaño de red. El aumento en los costos de los bancos, lo desplazan a un mayor precio por apertura de cuenta, sin embargo, el aumento en precios es menor para el banco con menor red de cajeros, lo que le permite aumentar su participación de mercado y sus ganancias.

El artículo se organiza de la siguiente manera: en la segunda sección se hace el planteamiento del modelo; en la tercera se presentan los resultados de equilibrio antes y después de la regulación; la sección cuatro presenta los efectos de la regulación haciendo la comparación de los equilibrios, y finalmente en la cinco se presentan las conclusiones.

\section{Modelo}

Se utiliza una modelación similar a la propuesta por Chioveanu, Fauli-Oller, Sandonis y Santamaría (2009). Bajo un esquema de competencia espacial de Hotelling, dos bancos se localizan en los extremos de una línea de tamaño unitario, éste es el espacio bancario. Los consumidores realizan compras y retiros de efectivo en otro espacio, que se define como espacio de compra. En el espacio de compra, a diferencia de Chioveanu et al, los cajeros no se localizan en tiendas comerciales, sino que los cajeros están uniformemente localizados en el espacio de compra. Este supuesto elimina la posibilidad de que no existan transacciones interbancarias dado que dentro del espacio de compra se localicen al mismo tiempo dos cajeros. Para poder analizar el efecto de la regulación, partiendo de asimetrías en el tamaño de red, se hace el supuesto de que el número de cajeros instalado por cada banco ya está determinado. Dada la complejidad de las ecuaciones se utilizan métodos numéricos para calcular el equilibrio; esto es, calcular el equilibrio para cada posible situación de asimetrías en el tamaño de red.

\section{II.1 Bancos}

Se considera un modelo con dos bancos (A y B). Los bancos están localizados en los extremos de un segmento de tamaño unitario, donde los consumidores están uniformemente distribuidos. Los bancos ofrecen dos servicios a sus usuarios: servicios de banca y servicios de cajeros. El número de cajeros instalados por el banco $i$ es exógeno y está dado por $n_{i}$. El tamaño total de red es $n=n_{A}+n_{B}$. Por 
simplicidad en el número de posibles escenarios se consideran solamente las siguientes situaciones $\left(n_{A}, n_{B}\right) \in\{(1,1),(2,1),(5,1),(1,0)\}$. Los costos fijos de instalar y operar un cajero automático se denotan por $c$, donde $c>0$. Se hace el supuesto de que los cajeros están uniformemente distribuidos.

Por tener una cuenta en el banco $i$ el banco cobra a sus usuarios $F_{i}$. Los costos para el banco de manejar una cuenta por simplicidad se normalizan a cero. Dado que la red de cajeros automáticos es compartida, esto les permite a los consumidores, de acuerdo a su conveniencia, realizar transacciones en cualquier cajero de la red, por lo que la transacción puede ser realizada tanto en un cajero propio como en un cajero ajeno. Los costos marginales de procesar una transacción en un cajero están denotados por $Z$, donde $z \geq 0$. Por simplicidad se hace el supuesto de que $z=0.2$. El cobro por transacciones en cajeros depende de la regulación vigente; por lo tanto, el artículo analiza dos escenarios diferentes en el cobro de transacciones en cajeros:

i) Escenario Base: si la transacción es realizada por usuarios propios, el banco $\boldsymbol{i}$ establece una comisión por el uso de su cajero $d_{i}$. Cuando un usuario del banco $i$ realiza una transacción en un cajero del banco $j$, el usuario tiene que pagar una cuota de sobrecargo $S_{j}$ al banco operador del cajero y una comisión por uso de cajero ajeno $f_{i}$ al banco al cual está afiliado; adicionalmente, el banco $\boldsymbol{i}$ paga una cuota de intercambio $\boldsymbol{a}$ al banco $\boldsymbol{j}$.

ii) Escenario Regulación Banco de México: si la transacción es realizada por usuarios propios, la transacción es libre de cargo. Si un usuario del banco $i$ realiza una transacción en un cajero del banco $j$, el usuario paga un cobro directo $S_{j r}$ al banco operador del cajero; adicionalmente, el banco $j$ paga una comisión $a_{I}$ al banco $\boldsymbol{i}$ (cuota de intercambio inversa).

Para evitar soluciones de esquina se hace el supuesto de que: $0 \leq a<1 \mathrm{y}$ $0<a_{I}+z<1$.

\section{II.2 Consumidores}

Existe un continuo de consumidores (de tamaño uno), los cuales deben elegir un banco. Los costos de transporte para el consumidor vienen dados por $C_{t}(x)=x$, donde $x$ denota la ubicación del consumidor respecto al banco $i$. Un consumidor que abre una cuenta con algún banco obtiene un nivel de utilidad bruta $M$, para la cual se hace el supuesto de que es lo suficientemente grande para garantizar una cobertura total en el mercado. 
Adicionalmente, se hace el supuesto de que cuando los consumidores necesitan hacer una transacción en cajeros, éstos realizan sus transacciones en otro espacio, el cual se denomina espacio de compra. En el espacio de compra los consumidores buscan el cajero y deciden si realizan la transacción en el primero que encuentran; se hace el supuesto que es altamente costoso cambiar de ubicación. Con una probabilidad $P_{i}$ un consumidor encuentra un cajero del banco al cual está afiliado y con una probabilidad $P_{j}$ encuentra un cajero ajeno. Dado que los cajeros están uniformemente distribuidos, estas probabilidades están determinadas por las densidades de cajeros, específicamente $P_{i}=n_{i} / n$ y $P_{j}=n_{j} / n{ }^{7}$ Bajo esta modelación las comisiones en cajeros no afectan la elección del cajero, pero sí el nivel en el que son usados. ${ }^{8}$

Los consumidores también pueden obtener utilidad al realizar transacciones en cajeros, esto debido a la posibilidad de retirar efectivo y obtener liquidez en los cajeros automáticos. El consumidor obtiene un beneficio bruto al utilizar un cajero igual a $v$, donde $v$ es una variable aleatoria uniformemente en el intervalo $[0,1]$. A continuación se presenta la estructura del juego:

\section{Etapas del juego}

i) Dada la cuota de intercambio, los bancos establecen de forma simultánea y no-cooperativa precios por apertura de cuenta $F_{i}$ y, dada la regulación vigente, determinan las comisiones por transacciones en cajeros.

ii) Los consumidores deciden a qué banco afiliarse observando el número de cajeros instalados por cada banco, precios y comisiones por transacciones en cajeros.

iii) En la última etapa los consumidores encuentran un cajero automático y dada la valoración $v$ deciden si lo usan o no.

En primera instancia se hace el supuesto de que la cuota de intercambio es conocida por los bancos y está exógenamente determinada; posteriormente se elimina este supuesto para comparar los resultados de la regulación. En las siguientes

${ }^{7}$ Dado que en la modelación lo que importa es la probabilidad de encontrar un cajero, los resultados de equilibrio son los mismos a los que obtendríamos si escalamos para todos los bancos el tamaño de red.

${ }^{8}$ Esto implica que la elasticidad de la demanda se reduce al elevar los costos de transporte. A diferencia de los modelos espaciales, la distancia en esta modelación no juega ningún papel en la demanda de servicios de cajeros. 
secciones se busca bajo cada posible escenario de regulación el equilibrio de Nash perfecto en sub-juegos mediante inducción hacia atrás. Dada la complejidad de las ecuaciones el equilibrio se aproxima de forma numérica para cada posible configuración.

\section{EQUILIBRIOS}

\section{III.1 Equilibrio del Escenario Base}

En la última etapa, con una probabilidad $P_{i}=n_{i} / n$ el consumidor encontrará un cajero del banco al cual está afiliado, sólo realizará la transacción si $v \geq d_{i}$. La probabilidad de que el usuario realice la transacción es $\left(1-d_{i}\right)$. Con una probabilidad $P_{j}=n_{j} / n$ el consumidor encontrará un cajero ajeno. Solamente utilizará el cajero ajeno si $v \geq f_{i}+s_{j}$, donde $f_{i}$ es la comisión por uso de cajero ajeno establecida por su banco emisor de la tarjeta y $s_{j}$ la cuota de sobrecargo cobrada por el operador del cajero. La probabilidad de que realice la transacción es $\left(1-f_{i}-s_{j}\right)$.

En la penúltima etapa los consumidores deciden a qué banco afiliarse, por lo cual tienen que comparar la utilidad esperada de afiliarse al banco A y B. Para un consumidor localizado a una distancia $\mathcal{X}$ éstas vienen dadas respectivamente por:

$$
\begin{aligned}
& M-x-F_{A}+P_{A} \frac{\left(1-d_{A}\right)^{2}}{2}+P_{B} \frac{\left(1-f_{A}-s_{B}\right)^{2}}{2} \\
& M-(1-x)-F_{B}+P_{B} \frac{\left(1-d_{B}\right)^{2}}{2}+P_{A} \frac{\left(1-f_{B}-s_{A}\right)^{2}}{2}
\end{aligned}
$$

Los primeros tres términos de las ecuaciones anteriores capturan la utilidad neta de los consumidores procedente de los servicios de banca básica por tener una cuenta bancaria. Por su parte, los últimos dos términos representan la utilidad neta esperada por los servicios de cajeros. Considere un consumidor del banco $i$; con una probabilidad $P_{i}=n_{i} / n$, localizará un cajero del banco al cual está afiliado, dado que el costo de la transacción es $d_{i}$, su utilidad neta esperada es $\frac{\left(1-d_{i}\right)^{2}}{2}$. Con una probabilidad $P_{j}=n_{j} / n$, encontrará un cajero ajeno; como el costo por transacciones interbancarias es $f_{i}+s_{j}$ su utilidad neta esperada por transacciones en cajeros ajenos es $\frac{\left(1-f_{i}-s_{j}\right)^{2}}{2}$. 
Igualando las ecuaciones (1) y (2) se obtiene la participación de mercado del banco A:

$$
x=\frac{1}{2}+\frac{F_{B}-F_{A}}{2}+\frac{P_{A}\left(1-d_{A}\right)^{2}-P_{B}\left(1-d_{B}\right)^{2}}{4}+\frac{P_{B}\left(1-f_{A}-s_{B}\right)^{2}-P_{A}\left(1-f_{B}-s_{A}\right)^{2}}{4}
$$

La expresión anterior captura cómo los cambios en precios y tamaño de red tienen impacto en la demanda del banco A; por ejemplo, una reducción en la comisión por uso de cajero ajeno $f_{A}$ y/o en la cuota de uso en cajeros propios $d_{A}$, genera que el banco A se vuelva más atractivo (efecto análogo a si el banco B incrementa estas comisiones). Otro aspecto interesante es que una disminución de la cuota de sobrecargo $S_{A}$ (manteniendo todo lo demás constante) por parte del banco A genera una pérdida de consumidores en favor del banco B, debido a que los usuarios prefieren afiliarse al banco B para evitar el cobro excesivo por transacciones interbancarias.

En la primera etapa del juego los bancos determinan precios por apertura de cuenta $F_{i}$, así como comisiones por uso en cajeros propios $\left(d_{i}\right.$ y $\left.s_{i}\right)$ y ajenos $\left(f_{i}\right)$ con el propósito de maximizar sus ganancias. Las ganancias del banco A están dadas por:

$$
\begin{aligned}
& \pi_{A}=x\left(F_{A}\right)+\pi_{A}^{A T M} \\
& \begin{array}{r}
\pi_{A}^{A T M}=x P_{A}\left(1-d_{A}\right)\left(d_{A}-z\right)+x P_{B}\left(1-f_{A}-s_{B}\right)\left(f_{A}-a\right) \\
\quad+(1-x) P_{A}\left(1-f_{B}-s_{A}\right)\left(s_{A}+a-z\right)-c n_{A}
\end{array}
\end{aligned}
$$

El primer término de la ecuación (4) representa los ingresos obtenidos por todos aquellos usuarios que abrieron una cuenta en el banco A. La ecuación (5) captura las ganancias por los servicios en cajeros, tanto de usuarios propios como ajenos. El primer término de la ecuación 5 expresa el ingreso procedente de los retiros de clientes propios en cajeros propios. El segundo término expresa el ingreso obtenido por los retiros de clientes propios en cajeros ajenos. Por su parte, el penúltimo término son las ganancias obtenidas por los retiros de clientes ajenos en cajeros propios. Finalmente $c n_{A}$ son los costos de instalación de $n_{A}$ cajeros automáticos.

La siguiente tabla muestra los valores de equilibrio para cada una de las siguientes situaciones $\left(n_{A}, n_{B}\right) \in\{(1,1),(2,1),(5,1),(1,0)\}$.

Equilibrios numéricos en el escenario base 
Tabla I

\begin{tabular}{|c|c|c|c|c|}
\hline & $n_{A}=1, n_{B}=1$ & $n_{A}=2, n_{B}=1$ & $n_{A}=5, n_{B}=1$ & $n_{A}=1, n_{B}=0$ \\
\hline $\boldsymbol{d}_{A}^{*}$ & 0.2 & 0.2 & 0.2 & 0.2 \\
\hline$d_{B}^{*}$ & 0.2 & 0.2 & 0.2 & --- \\
\hline $\boldsymbol{f}_{A}^{*}$ & a & a & a & --- \\
\hline$f_{B}^{*}$ & a & a & a & a \\
\hline $\boldsymbol{s}_{A}^{*}$ & $0.73334-a$ & $0.73625-a$ & $0.73922-a$ & $0.74216-a$ \\
\hline$s_{B}^{*}$ & $0.73334-a$ & $0.73045-a$ & $0.72759-a$ & --- \\
\hline$F_{A}^{*}$ & 1.0711 & 1.1106 & 1.1498 & 1.1888 \\
\hline$F_{B}^{*}$ & 1.0711 & 1.0313 & 0.9913 & 0.95101 \\
\hline $\boldsymbol{x}$ & 0.5 & 0.50814 & 0.51634 & 0.52448 \\
\hline$\pi_{A}^{*}$ & $0.57111-c$ & $0.61072-2 c$ & $0.65036-5 c$ & $0.68998-c$ \\
\hline$\pi_{B}^{*}$ & $0.57111-c$ & $0.53147-c$ & $0.49182-c$ & 0.45222 \\
\hline$C S^{*}$ & $M-1.1433$ & $M-1.1432$ & $M-1.143$ & $M-1.1427$ \\
\hline
\end{tabular}

Demostración: Ver Apéndice I.1.

\section{Resultados}

En lo que respecta al escenario $\left(n_{A}, n_{B}\right)=(1,0)$ se ve de forma clara que el banco B no puede cobrar cuotas de sobrecargo ni comisiones por uso de cajeros propios, ya que no cuenta con cajeros y, por lo tanto, el banco A está imposibilitado de cobrar comisiones por uso de cajero ajeno.

Como se puede observar, el tamaño de red de cada banco tiene efectos directos sobre su participación de mercado. El resultado es congruente con estudios empíricos como el obtenido por Massoud, Saunders y Scholnick (2005), donde encontraron que una mayor red de cajeros permite a los bancos captar mayores participaciones de mercado.

Analizando los equilibrios se observan correlaciones positivas entre un mayor tamaño de red con cuotas de sobrecargo $\left(s_{i}^{*}\right)$, precios por apertura de cuenta $\left(F_{i}^{*}\right)$, y ganancias de los bancos $\left(\pi_{i}^{*}\right)$. Comparando cada uno de los escenarios, de izquierda a derecha (lo cual implica una mayor asimetría en tamaño de red), se puede observar el incremento para el banco A en cada una de las variables, así como la disminución de cada una de ellas para el banco B. ${ }^{9}$

En equilibrio, el precio por apertura de cuenta está compuesto por el parámetro de diferenciación en el espacio (esto es, 1), el costo de oportunidad para el banco $\boldsymbol{i}$ de atraer un nuevo consumidor y por el grado de diferenciación en el tamaño de red. Por ejemplo: en el caso $\left(n_{A}, n_{B}\right)=(1,1)$, donde no existe diferencia en el tamaño de red, el precio por apertura de cuenta es la suma del parámetro de

\footnotetext{
${ }^{9}$ Resultados obtenidos por Chioveanu, Fauli-Oller, Sandonis y Santamaría (2009).
} 
diferenciación más el costo de oportunidad. El costo de oportunidad está dado por: $P_{i}\left(1-f_{j}-s_{i}\right)\left(s_{i}+a-z\right)$. Con un probabilidad $P_{i}$ los usuarios del banco $j$ hubieran encontrado cajeros del banco $i$, realizando esa transacción con una probabilidad igual a $\left(1-f_{j}-s_{i}\right)$, representando una ganancia para el banco $i$ de $\left(s_{i}+a-z\right)$. Sustituyendo los valores de equilibrio tenemos que el costo de oportunidad de atraer un nuevo consumidor es 0.0711 .

Los bancos establecen a sus usuarios, tanto por transacciones en cajeros propios como ajenos, comisiones iguales al costo marginal $d_{i}^{*}=z, f_{i}^{*}=a$. Con el propósito de atraer clientes de la competencia, al incrementar el costo por transacciones interbancarias, los bancos establecen la cuota de sobrecargo $s_{i}$ a un nivel superior al que maximiza sus ingresos por transacciones interbancarias. ${ }^{10}$

Finalmente, un resultado relevante es que las variables de mercado tales como ganancias de los bancos, excedente del consumidor, participación de mercado y precios por apertura de cuenta no dependen del valor de la cuota de intercambio. La razón de lo anterior es que un aumento de la cuota de intercambio genera un aumento de la misma cuantía en la comisión por uso de cajero ajeno, así como una reducción del mismo monto en la cuota de sobrecargo, por lo que su efecto es totalmente compensado.

En la siguiente sección se analiza el equilibrio resultante bajo las condiciones establecidas después de la regulación.

\section{III.2 Equilibrio del escenario regulación Banco de México}

En la última etapa, con una probabilidad $P_{i}=n_{i} / n$ el consumidor encontrará un cajero del banco al cual está afiliado, dado que la transacción es libre de cargo la probabilidad de que la realice es uno. Con una probabilidad $P_{j}=n_{j} / n$ el consumidor encontrará un cajero ajeno, sólo utilizará el cajero si $v \geq s_{j r}$, donde $s_{j r}$ representa el cobro directo establecido por el operador del cajero. La probabilidad de que realice la transacción es $\left(1-s_{j r}\right)$.

En la penúltima etapa los consumidores deciden a qué banco afiliarse, por lo cual tienen que comparar la utilidad esperada de afiliarse al banco A y B. Para un consumidor localizado a una distancia $\boldsymbol{X}$ éstas vienen dadas respectivamente por:

$M-x-F_{A r}+P_{A} \frac{1}{2}+P_{B} \frac{\left(1-s_{B r}\right)^{2}}{2}$

\footnotetext{
${ }^{10}$ Esto es, $0.5+0.5 z-a$
} 
$M-(1-x)-F_{B r}+P_{B} \frac{1}{2}+P_{A} \frac{\left(1-s_{A r}\right)^{2}}{2}$

Siguiendo la misma lógica respecto a la situación original, igualando las ecuaciones anteriores se obtiene la participación de mercado para el banco A:

$x=\frac{1}{2}+\frac{F_{B r}-F_{A r}}{2}+\frac{P_{A}-P_{B}}{4}+\frac{P_{B}\left(1-s_{B r}\right)^{2}-P_{A}\left(1-s_{A r}\right)^{2}}{4}$

Dado que las transacciones en cajeros propios son libres de costo, en la expresión anterior fácilmente se puede observar que la demanda del banco A se incrementa a medida que aumenta $P_{A}-P_{B} \cdot{ }^{11}$ La razón es que los consumidores prefieren afiliarse al banco que les permita hacer un mayor número de transacciones en cajeros libres de costo, que realizar transacciones en cajeros ajenos a costo $S_{i r}$.

Bajo este escenario las ganancias del banco A vienen dadas por:

$$
\begin{aligned}
& \pi_{A r}=x\left(F_{A r}\right)+\pi_{A}^{A T M(r)} \\
& \pi_{A}^{A T M(r)}=x P_{A}(-z)+x P_{B}\left(1-s_{B r}\right)\left(a_{I}\right)+(1-x) P_{A}\left(1-s_{A r}\right)\left(s_{A r}-a_{I}-z\right)-c n_{A}
\end{aligned}
$$

A diferencia del escenario base, dado que la regulación estableció que las transacciones en cajeros propios fueran libres de costo, el término $x P_{A}(-z)$ representa los costos de procesar los retiros de clientes propios en cajeros propios. Por su parte $x P_{B}\left(1-s_{B}^{r}\right)\left(a_{I}\right)$ representa las ganancias obtenidas por todas aquellas transacciones de usuarios propios en cajeros ajenos. El penúltimo término $(1-x) P_{A}\left(1-s_{A r}\right)\left(s_{A r}-a_{I}-z\right)$ expresa los ingresos procedentes de retiros de clientes ajenos en cajeros propios. Finalmente $c n_{A}$ son los costos de instalación de la red de cajeros por parte del banco A. Como se puede observar ahora el banco operador, del cajero paga la cuota de intercambio $a_{I}$ al banco emisor de la tarjeta y se elimina el pago de comisiones por uso de cajero ajeno.

Las siguientes tablas muestran los valores de equilibrio para cada una de las siguientes situaciones $\left(n_{A}, n_{B}\right) \in\{(1,1),(2,1),(5,1),(1,0)\}$. A diferencia del escenario base, el equilibrio depende del valor de la cuota de intercambio inversa; para reducir el número de escenarios posibles, se calculan los equilibrios para tres valores distintos de $a_{I} \in\{(0),(0.5),(0.7)\}$.

Equilibrios numéricos en el escenario con regulación

${ }^{11}$ Dado que $P_{i}=n_{i} / n$ esta diferencia captura las asimetrías en el tamaño de red entre los bancos. 
Tabla II

\begin{tabular}{|l|c|c|c|c|}
\hline $\boldsymbol{a}_{\boldsymbol{I}}=\mathbf{0}$ & $\boldsymbol{n}_{\boldsymbol{A}}=\mathbf{1}, \boldsymbol{n}_{\boldsymbol{B}}=\mathbf{1}$ & $\boldsymbol{n}_{\boldsymbol{A}}=\mathbf{2}, \boldsymbol{n}_{\boldsymbol{B}}=\mathbf{1}$ & $\boldsymbol{n}_{\boldsymbol{A}}=\mathbf{5}, \boldsymbol{n}_{\boldsymbol{B}}=\mathbf{1}$ & $\boldsymbol{n}_{\boldsymbol{A}}=\mathbf{1}, \boldsymbol{n}_{\boldsymbol{B}}=\mathbf{0}$ \\
\hline $\boldsymbol{s}_{\boldsymbol{A r}}^{*}$ & 0.73334 & 0.73583 & 0.73835 & 0.74094 \\
\hline $\boldsymbol{s}_{\boldsymbol{B r}}^{*}$ & 0.73334 & 0.73086 & 0.72839 & -- \\
\hline $\boldsymbol{F}_{\boldsymbol{A r}}^{*}$ & 1.1711 & 1.2417 & 1.3121 & 1.3822 \\
\hline $\boldsymbol{F}_{\boldsymbol{B} \boldsymbol{r}}^{*}$ & 1.1711 & 1.1003 & 1.0292 & 0.9579 \\
\hline $\boldsymbol{X}$ & 0.5 & 0.50704 & 0.51403 & 0.52107 \\
\hline $\boldsymbol{\pi}_{\boldsymbol{A r}}^{*}$ & $0.57111-c$ & $0.60851-2 c$ & $0.64583-5 c$ & $0.68312-c$ \\
\hline $\boldsymbol{\pi}_{\boldsymbol{B r}}^{*}$ & $0.57111-c$ & $0.53369-c$ & $0.49626-c$ & 0.45877 \\
\hline $\boldsymbol{C} \boldsymbol{S}_{\boldsymbol{r}}^{*}$ & $M-1.1533$ & $M-1.1533$ & $M-1.1531$ & $M-1.1528$ \\
\hline
\end{tabular}

Tabla III

\begin{tabular}{|l|c|c|c|c|}
\hline $\boldsymbol{a}_{\boldsymbol{I}}=\mathbf{0 . 5}$ & $\boldsymbol{n}_{\boldsymbol{A}}=\mathbf{1}, \boldsymbol{n}_{\boldsymbol{B}}=\mathbf{1}$ & $\boldsymbol{n}_{\boldsymbol{A}}=\mathbf{2}, \boldsymbol{n}_{\boldsymbol{B}}=\mathbf{1}$ & $\boldsymbol{n}_{\boldsymbol{A}}=\mathbf{5}, \boldsymbol{n}_{\boldsymbol{B}}=\mathbf{1}$ & $\boldsymbol{n}_{\boldsymbol{A}}=\mathbf{1}, \boldsymbol{n}_{\boldsymbol{B}}=\mathbf{0}$ \\
\hline $\boldsymbol{s}_{\boldsymbol{A r}}^{*}$ & 0.9 & 0.90171 & 0.90344 & 0.90519 \\
\hline $\boldsymbol{s}_{\boldsymbol{B r}}^{*}$ & 0.9 & 0.89832 & 0.89668 & -- \\
\hline $\boldsymbol{F}_{\boldsymbol{A r}}^{*}$ & 1.085 & 1.155 & 1.2252 & 1.2957 \\
\hline $\boldsymbol{F}_{\boldsymbol{B} \boldsymbol{r}}^{*}$ & 1.085 & 1.0152 & 0.94569 & 0.87638 \\
\hline $\boldsymbol{X}$ & 0.5 & 0.51268 & 0.52541 & 0.53809 \\
\hline $\boldsymbol{\pi}_{\boldsymbol{A r}}^{*}$ & $0.51-c$ & $0.53892-2 c$ & $0.56846-5 c$ & $0.59857-c$ \\
\hline $\boldsymbol{\pi}_{\boldsymbol{B} \boldsymbol{r}}^{*}$ & $0.51-C$ & $0.48165-c$ & $0.45387-c$ & 0.42671 \\
\hline $\boldsymbol{C}_{\boldsymbol{r}}^{*}$ & $M-1.0825$ & $M-1.0825$ & $M-1.0824$ & $M-1.0823$ \\
\hline
\end{tabular}

Tabla IV

\begin{tabular}{|c|c|c|c|c|}
\hline$a_{I}=0.7$ & $n_{A}=1, n_{B}=1$ & $n_{A}=2, n_{B}=1$ & $n_{A}=5, n_{B}=1$ & $n_{A}=1, n_{B}=0$ \\
\hline $\boldsymbol{s}_{A r}^{*}$ & 0.96667 & 0.96735 & 0.9680 & 0.96877 \\
\hline$s_{B r}^{*}$ & 0.96667 & 0.96598 & 0.96534 & --- \\
\hline $\boldsymbol{F}_{A r}^{*}$ & 1.0894 & 1.1575 & 1.2256 & 1.2940 \\
\hline$F_{B r}^{*}$ & 1.0894 & 1.0216 & 0.95384 & 0.8863 \\
\hline$x$ & 0.5 & 0.5153 & 0.53062 & 0.54591 \\
\hline$\pi_{A r}^{*}$ & 0.50109-c & $0.53255-2 c$ & $0.56489-5 c$ & $0.5982-c$ \\
\hline$\pi_{B r}^{*}$ & c & $0.47063-c$ & $0.44103-c$ & 0.41239 \\
\hline$C S^{*}$ & $M-1.0891$ & $M-1.089$ & $M-1.0885$ & $M-1.0878$ \\
\hline
\end{tabular}

Demostración: Ver Apéndice 1.2. 


\section{Resultados}

Como se podría esperar, al igual que el escenario base, se observan para cada uno de los escenarios correlaciones positivas entre un mayor tamaño de red con participaciones de mercado, cobro directo, precios por apertura de cuenta y ganancias de los bancos.

En este nuevo escenario, a diferencia del escenario base, la cuota de intercambio inversa $a_{I}$ es relevante en la determinación de todas las variables del modelo; esto se debe a que se pierde su neutralidad dada la desaparición de las comisiones por uso de cajero ajeno. Por otro lado, el cobro directo $S_{\text {ir }}$ se establece a un nivel superior al que maximiza los ingresos por transacciones interbancarias (esto es, $0.5+0.5 z+0.5 a_{I}$ ). Por lo tanto, el cobro directo es utilizado no sólo para obtener ganancias por las transacciones en cajeros, sino también como un instrumento estratégico para obtener mayores participaciones de mercado.

Un aspecto interesante del equilibrio es que los bancos con menores redes de cajeros establecen $S_{j r}$ a niveles muy similares al de bancos con mayores redes de cajeros. Establecer comisiones menores por parte del banco pequeño hace más atractivo para los consumidores afiliarse al banco que les cobre una comisión mayor. Afiliándose al banco grande los consumidores evitan el cobro excesivo por transacciones interbancarias y realizan estas transacciones en cajeros del banco pequeño que les cobra una comisión menor.

En equilibrio, al igual que el escenario base, el precio por apertura de cuenta está compuesto por el parámetro de diferenciación en el espacio, el costo de oportunidad para el banco $\boldsymbol{i}$ de atraer un nuevo consumidor y por el grado de diferenciación en el tamaño de red. En este caso el costo de oportunidad está dado por: $P_{i}\left(1-s_{i r}\right)\left(s_{i r}-a_{I}-z\right)+P_{i}(z)-P_{j}\left(1-s_{j r}\right)\left(a_{I}\right)$. Con una probabilidad $P_{i}$ el usuario del banco $j$ hubiera encontrado cajeros del banco $i$ realizando esa transacción con una probabilidad igual a $\left(1-s_{i r}\right)$, reportándole un ingreso de $\left(s_{i r}-a_{I}-z\right)$. Afiliado al banco $i$ este usuario encontrará cajeros propios con una probabilidad $P_{i}$ generando un costo de $(z)$. Finalmente, dicho usuario con una probabilidad $P_{j}$

encontrará cajeros del banco al cual estaba afiliado, con probabilidad $\left(1-s_{j r}\right)$ realizará la transacción reportando una ganancia neta al banco $i$ de $a_{I}$.

\section{EFectos de la Regulación}

Hasta este momento el análisis se había desarrollado suponiendo exógena la cuota de intercambio (tradicional e inversa). En esta sección, se amplía el análisis incluyendo una etapa adicional al juego (etapa 0). En esta etapa se hace el supuesto de 
que los bancos seleccionan cooperativamente la cuota de intercambio con el propósito de maximizar sus ganancias conjuntas. ${ }^{12}$

En el escenario con regulación se puede ver claramente que las ganancias de los bancos son decrecientes en $a_{I}$, por lo que si los bancos seleccionan cooperativamente su valor para maximizar sus ganancias conjuntas, la elección es $a_{I}=0 .{ }^{13}$ En el escenario base las ganancias de los bancos son neutrales al valor de la cuota de intercambio, por lo que la decisión de $\boldsymbol{a}$ es irrelevante. Estos resultados permiten analizar los efectos de la regulación bajo situaciones asimétricas en el tamaño de red; comparando el escenario base respecto al escenario con regulación cuando $a_{I}=0$.

Bajo esta situación, comparando los resultados de la tabla I y II, la regulación genera los siguientes resultados. En primera instancia, a pesar de que la regulación eliminó el cobro de comisiones por uso de cajero ajeno, el precio por realizar transacciones en cajeros distintos al del banco emisor de la tarjeta prácticamente se mantuvo sin cambios $f_{i}^{*}+s_{j}^{*} \approx s_{j r}^{*}{ }^{14}$ Por lo tanto, sin importar asimetrías en el tamaño de red, la regulación no disminuye el cobro por transacciones interbancarias. Al igual que Kaiser y Lever (2011), se observa un desplazamiento de las comisiones por el uso de cajeros propios, las cuales la regulación prohíbe, a mayores precios por apertura de cuenta. La regulación, al establecer que las transacciones de usuarios propios en cajeros propios fueran gratuitas, genera un aumento en los costos del banco $\boldsymbol{i}$ de $P_{i}(-z) .{ }^{15} \mathrm{El}$ banco transfiere este aumento en costos a un mayor precio de apertura de cuenta. Por ejemplo, analizando para $\left(n_{A}, n_{B}\right)=(2,1)$ el aumento en costos para el banco A está dado por $P_{i} z=\frac{2}{3}(0.2)=0.1333$. Sin embargo, por cuestiones de demanda, el aumento en precios por apertura de cuenta es un poco menor $F_{i r}^{*}-F_{i}^{*}=1.2417-1.1106=0.1311$. Para el caso simétrico la transferencia es total.

Al contrario de lo que se podría esperar, la regulación favorece a los bancos pequeños. Comparando las ganancias de los bancos, antes y después de la regulación, se observa que las ganancias del banco con un mayor número de cajeros instalados disminuyen mientras que las ganancias del banco con menos cajeros aumentan. La explicación subyace en los precios por apertura de cuenta. Dado que

${ }^{12}$ La idea de seleccionar cooperativamente la cuota de intercambio es un supuesto común en la literatura, por ejemplo, Donze y Dubec (2009), Fauli-Oller (2008), Matutes y Padilla (1994), entre otros, lo cual es congruente con las prácticas reales de su determinación.

${ }^{13}$ Esta conclusión se cumple para los diferentes valores $a_{I}$ bajo el supuesto de que $a_{I}+z<1$.

${ }^{14}$ Para el caso simétrico las comisiones por transacciones interbancarias son iguales antes y después de la regulación.

${ }^{15}$ Un usuario del banco $i$ con una probabilidad $P_{i}$ encontrará cajeros locales, generando un costo por transacción de $-z$. 
las transacciones de usuarios propios en cajeros propios son gratuitas, el aumento en $\operatorname{costos} P_{i} Z$ es mayor para el banco con un mayor tamaño de red. Los bancos desplazan el aumento en costos a un mayor precio por apertura de cuenta; sin embargo, el aumento en precios es menor para el banco con menos cajeros, lo que le permite aumentar su participación de mercado y sus ganancias. ${ }^{16}$ En conclusión, a la luz de los resultados obtenidos bajo un escenario asimétrico, la regulación favorece la competencia entre los bancos.

De acuerdo con Kaiser y Lever (2011), prohibir las comisiones a cuentahabientes también reduce la cuota de sobrecargo, pero aumenta los precios por apertura de cuenta. El aumento en precios por apertura de cuenta es menor que la disminución en las otras comisiones provocando un aumento en el excedente de los consumidores. A diferencia del resultado obtenido por Kaiser y Lever (2011), se encuentra que el aumento en precios por apertura de cuenta excede al beneficio obtenido por transacciones gratuitas en cajeros propios, lo que ocasiona una disminución en el excedente de los consumidores.

Finalmente, analizando las diferentes situaciones $\left(a_{I} \in\{(0),(0.5),(0.7)\}\right)$, en el escenario con regulación se observa que un aumento de la cuota de intercambio inversa disminuye las ganancias de los bancos, pero incrementa el excedente de los consumidores, por lo que la determinación de la cuota de intercambio inversa no debe ser vista como una simple transferencia entre bancos, sino que puede servir como un mecanismo para balancear entre ganancias de los bancos y excedente de los consumidores.

\section{Conclusiones}

Las comisiones cobradas por transacciones en cajeros han generado toda una serie de controversias en varios países, debido principalmente a que el cobro de estas comisiones es poco transparente en cuanto a su determinación. A raíz de esto, se han generado una serie de regulaciones con el propósito de mejorar la competencia y la eficiencia en el mercado de cajeros. En México, a partir de mayo de 2010 entró en vigor una reforma desarrollada por el Banco de México, la cual permite dar transparencia en el cobro de comisiones, de tal manera que los usuarios conozcan antes de realizar la transacción el cobro por la misma. Adicionalmente, prohíbe el cobro de comisiones por uso de cajero ajeno y sólo permite la existencia de cobro directo por parte del operador del cajero.

${ }^{16}$ Adicionalmente, la demanda es más sensible ante variaciones en precios por apertura de cuenta que a variaciones en comisiones en cajeros; por lo que, el aumento en precios por apertura de cuenta más que compensa las diferencias en tamaños de red. 
La presente investigación se enfocó en analizar los efectos de la regulación establecida por el Banco de México en 2010, desde una perspectiva teórica. Partiendo de un escenario asimétrico en el tamaño de red, se analizó el efecto de la regulación sobre aquellos bancos con redes de cajeros pequeñas y, por lo tanto, mayores proporciones de retiros de clientes propios en cajeros ajenos, los cuales parecían ser los principales afectados con el nuevo esquema de comisiones al no poder compensar a sus usuarios por las transacciones en cajeros ajenos.

Los resultados sugieren que a pesar de que la regulación elimina el cobro de comisiones por uso de cajero ajeno, con el propósito de disminuir el cobro excesivo por transacciones interbancarias, la comisión por transacciones interbancarias se mantiene relativamente sin cambios. Adicionalmente, se observa que se reducen las comisiones por el uso de cajeros propios a cambio de mayores precios por apertura de cuenta, lo cual sucede porque los usuarios son menos sustitutos entre sí al incrementarse los costos de oportunidad de atraer un nuevo consumidor. Se observa además que en términos del excedente del consumidor el aumento en precios por apertura de cuenta excede al beneficio obtenido por transacciones en cajeros propios gratuitas, lo que ocasiona una disminución en el excedente de los consumidores.

Finalmente, al contrario de lo que se podría esperar, la regulación favorece a los bancos pequeños. Se observa que la regulación favorece la competencia al generar que el aumento en costos, por la eliminación del cobro a usuarios propios en cajeros propios, sea mayor para el banco con un mayor tamaño de red. El aumento en costos los bancos lo desplazan a un mayor precio por apertura de cuenta, sin embargo, el incremento en precios es menor para el banco con menos cajeros, lo que le permite aumentar su participación de mercado y sus ganancias.

Sin embargo, para analizar las consecuencias que puede provocar la reforma sobre los bancos pequeños, se debe continuar el análisis de diferentes cuestiones, tanto desde una perspectiva teórica y empírica. Por ejemplo: una consideración importante que en el modelo no está incluida y puede ser considerada para trabajos futuros es endogeneizar la relación entre costos marginales por transacción y tamaño de red. Se esperaría que el banco con mayor número de cajeros enfrente economías de escala y costos marginales por transacción menores en comparación con un banco con menos número de cajeros. Situación que a luz de los resultados obtenidos es relevante para entender los efectos reales de la regulación. 


\section{REFERENCIAS BIBLIOGRÁFICAS}

Banco de México (2010), Reporte sobre el Sistema Financiero.

Chioveanu, Ioana; Fauli-Oller, Ramón; Sandonis, Joel y Santamaría, Juana (2009), "Aтм Surcharges: Effects on Deployment and Welfare", The Journal of Industrial Economics, 57 (3), pp. 613-635.

Croft, Elizabeth y Spencer, Barbara (2004), "Fees and Surcharging in Automatic-Teller Machines Networks: Non-Bank ATM Providers versus Large Bank", NBER Working Paper w9883.

Diario Oficial de la Federación de fecha lunes 26 de julio de 2010, "Circular 14/2010 y 22/2010" dirigida a las instituciones de crédito, sociedades financieras de objeto limitado y sociedades financieras de objeto múltiple reguladas.

Donze, Jocelyn y Dubec, Isabelle (2006), "The Role of Interchange Fees in ATM Networks. International", Journal of Industrial Organization, 24 (1), pp. 29-43.

(2008), "The Effects of Regulating Interchange Fees at Cost on the ATM Market", mimeo, Working Paper.

(2009), "Paying for ATM usage: good for consumers, bad for Banks?", The Journal of Industrial Economics, 57 (3), pp. 583-612.

Economides, Nicholas y Salop, Steven (1992), "Competition and integration among Complements, and Network Market Structure", Journal of Industrial Economics, 40, pp. 105-130.

Fauli-Oller, Ramón (2008) "The Role of Interchange Fee on the Effect of Forbidding Price Discrimination of ATM Services", Working Paper.

González, Pedro y Plata, Leobardo (2015), "Análisis teórico de las modificaciones a la regulación de comisiones interbancarias en cajeros automáticos en México", Estudios Económicos, vol. (30), núm. 1, pp. 141-178.

Hannan, Timothy; Kiser, Elizabeth; Prager, Robin y McAndrews, James (2003), "To Surcharge or Not to Surcharge: An Empirical Investigation of ATM Pricing", The Review of Economics and Statistics, 85 (4), pp. 990-1002.

Kaiser, Karen y Lever, Carlos (2011), "Regulación de comisiones de cajero en un modelo de competencia espacial", Banco de México. Documentos de Investigación, núm. 2011-09.

Ley para la Transparencia y Ordenamiento de los Servicios Financieros (LTOSF) Nueva ley publicada en el Diario Oficial de la Federación el 15 de junio de 2007, última reforma publicada: DOF 25-05-2010. 
Massoud, Nadia y Bernhardt, Dan (2002), "Rip-off ATM Surcharges", Rand Journal of Economics, 33 (1), pp. 96-115.

Massoud, Nadia, Saunders, Anthony y Scholnick, Barry (2005), "The Impact of ATM surcharges on Large Versus Small Banks: Is There a Switching Effect?", Journal of Business forthcoming.

Matutes, Carmen y Padilla, Jorge (1994), "Shared ATM Networks and Banking Competition", European Economic Review, 38, pp. 1113-1138.

Prager, Robin (2001), "The Effects of ATM Surcharges on Small Banking Organizations", Review of Industrial Organization, vol. 18, núm. 2, pp. 161-173. 


\section{APÉNDICE}

\section{I.1 Escenario base:}

La matriz Hessiana de la función objetivo del escenario base.

$$
H=\left(\begin{array}{llll}
\frac{\partial^{2} \pi_{i}}{\partial F_{i}^{2}} & \frac{\partial^{2} \pi_{i}}{\partial F_{i} d_{i}} & \frac{\partial^{2} \pi_{i}}{\partial F_{i} f_{i}} & \frac{\partial^{2} \pi_{i}}{\partial F_{i} s_{i}} \\
\frac{\partial^{2} \pi_{i}}{\partial d_{i} F_{i}} & \frac{\partial^{2} \pi_{i}}{\partial d_{i}^{2}} & \frac{\partial^{2} \pi_{i}}{\partial d_{i} f_{i}} & \frac{\partial^{2} \pi_{i}}{\partial d_{i} s_{i}} \\
\frac{\partial^{2} \pi_{i}}{\partial f_{i} F_{i}} & \frac{\partial^{2} \pi_{i}}{\partial f_{i} d_{i}} & \frac{\partial^{2} \pi_{i}}{\partial f_{i}^{2}} & \frac{\partial^{2} \pi_{i}}{\partial f_{i} s_{i}} \\
\frac{\partial^{2} \pi_{i}}{\partial s_{i} F_{i}} & \frac{\partial^{2} \pi_{i}}{\partial s_{i} d_{i}} & \frac{\partial^{2} \pi_{i}}{\partial s_{i} f_{i}} & \frac{\partial^{2} \pi_{i}}{\partial s_{i}^{2}}
\end{array}\right)
$$

Para el caso $\left(n_{A}, n_{B}\right)=(2,1)$ la matriz Hessiana de segundas derivadas de la función objetivo debe ser negativa definida. La matriz (valuada en equilibrio) está dada por:

\section{Para el Banco A:}

$$
\begin{aligned}
& H(\text { equilibrio }) \\
& =\left(\begin{array}{cccc}
-1 & -0.53333 & -0.08985 & -2.9167 \times 10^{-3} \\
-0.53333 & -0.6232 & -0.04792 & -1.5556 \times 10^{-3} \\
-0.08985 & -0.04792 & -0.17745 & -2.6206 \times 10^{-4} \\
-2.9167 \times 10^{-3} & -1.5556 \times 10^{-3} & -2.6206 \times 10^{-4} & -0.96263
\end{array}\right) \\
& \operatorname{DET}\left(H_{11}\right)=-1<0 \\
& \operatorname{DET}\left(H_{22}\right)=0.33876>0 \\
& \operatorname{DET}\left(H_{33}\right)=-5.7378 \times 10^{-2}<0 \\
& \operatorname{DET}\left(H_{44}\right)=5.5233 \times 10^{-2}>0
\end{aligned}
$$


Para el Banco B:

$H$ (equilibrio)

$=\left(\begin{array}{cccc}-1 & -0.26667 & -0.17583 & -1.4417 \times 10^{-3} \\ -0.26667 & -0.23508 & -4.6889 \times 10^{-2} & -3.8444 \times 10^{-4} \\ -0.17583 & -4.6889 \times 10^{-2} & -0.35885 & 2.5349 \times 10^{-4} \\ -1.4417 \times 10^{-3} & -3.8444 \times 10^{-4} & 2.5349 \times 10^{-4} & -0.49489\end{array}\right)$

$\operatorname{DET}\left(H_{11}\right)=-1<0$

$\operatorname{DET}\left(H_{22}\right)=0.16397>0$

$\operatorname{DET}\left(H_{33}\right)=-0.05377<0$

$\operatorname{DET}\left(H_{44}\right)=0.02661>0$

Siguiendo la misma lógica se pueden obtener las matrices Hessianas para los casos $\left(n_{A}, n_{B}\right)=(5,1)$ y $\left(n_{A}, n_{B}\right)=(1,0)$. Es fácil verificar que en cada uno de los casos la matriz Hessiana es negativa definida.

\section{I.2 Escenario con regulación:}

La matriz Hessiana de la función objetivo del escenario con regulación.

$$
H=\left(\begin{array}{cc}
\frac{\partial^{2} \pi_{i r}}{\partial F_{i r}^{2}} & \frac{\partial^{2} \pi_{i r}}{\partial F_{i r} s_{i r}} \\
\frac{\partial^{2} \pi_{i r}}{\partial s_{i r} F_{i r}} & \frac{\partial^{2} \pi_{i r}}{\partial s_{i r}^{2}}
\end{array}\right)
$$

Para el caso $\left(n_{A}, n_{B}\right)=(5,1)$ y $a_{I}=0$ la matriz Hessiana de segundas derivadas de la función objetivo debe ser negativa definida. La matriz (valuada en equilibrio) está dada por:

Para el Banco A:

$H($ equilibrio $)=\left(\begin{array}{cc}-1 & -0.00628 \\ -0.00628 & -1.1881\end{array}\right)$ 
190 ECONOMÍA TeORÍA y PrÁctica • Nueva Época, número 47, julio-diciembre 2017

$$
\begin{aligned}
& \operatorname{DET}\left(H_{11}\right)=-1<0 \\
& \operatorname{DET}\left(H_{22}\right)=1.1881>0
\end{aligned}
$$

Para el Banco B:

$H($ equilibrio $)=\left(\begin{array}{cc}-1 & 0.00123 \\ 0.00123 & -0.2504\end{array}\right)$

$\operatorname{DET}\left(H_{11}\right)=-1<0$

$\operatorname{DET}\left(H_{22}\right)=0.2504>0$

Obteniendo las matrices Hessianas para los casos $a_{I} \in\{(0.5),(0.7)\}$ es fácil verificar que para cada situación son negativas definidas. La misma lógica aplica para las situaciones $\left(n_{A}, n_{B}\right)=(2,1)$ y $\left(n_{A}, n_{B}\right)=(1,0)$. 